\title{
Préparer le futur : responsabilité de la science et des scientifiques. Quelques repères
}

\author{
Paul Henri Bourrelier
}

\section{Un danger à éviter}

II faut d'abord rappeler que, de longue date, les historiens ont renoncé à toute prétention au déterminisme historique. Les scientifiques (surtout des sciences - dures *), du fait qu'ils étudient des lois déterministes, peuvent être tentés d'établir une prévision d'évolution ; c'est une tentation qu'il leur faut catégoriquement rejeter.

Le perfectionnement des modèles, la puissance croissante des moyens de traitement nourrissent de telles tentations : le passage du modèle analytique, explicatif, explorateur des scénarios à un modèle prédictif se fait confusément, et les mises en garde contre une exploitation abusive ne sont pas toujours assez nettes (elles peuvent être maladroites pour celui qui a besoin de crédits).

Ce danger d'intégrisme scientifique, conscient ou pas, est un danger majeur dans la mesure où il constitue un abus de confiance, une sorte d'usage terroriste de moyens intellectuels ; je le considère comme fondamentalement attentatoire à la liberté de la nature et de l'homme, et d'autant plus redoutable qu'il est pavé des meilleures intentions (voir l'intégrisme écologique et ses dérives).

La première responsabilité des scientifiques relève donc d'un principe d'abstention et de mesure

\section{Trois responsabilités positives}

La science et les scientifiques peuvent, à mon sens, apporter trois contributions importantes à l'affrontement du futur :

i) En familiarisant la société avec le traitement de l'incertitude. L'incertitude est un concept fondamental en science, elle est beaucoup moins présente et travaillée dans les comportements quotidiens, dans l'exercice du pouvoir ; on comprend bien pourquoi, puisque l'incertitude peut être un poison pour l'action. Les scientifiques doivent donc être des professeurs en incertitude, et des pourvoyeurs de techniques de traitement des situations d'incertitude. Leur responsabilité s'étend depuis la production d'une véritable méthode, c'est-àdire une métrologie qui ne cède pas au vertige analytique et qui n'ignore pas les marges d'erreurs de la chaîne d'observation, jusqu'aux concepts tels que ceux des théories particulaires, biologiques, astronomiques et de celle, à l'échelle médiane, du chaos. II y a un important effort d'unification, d'approfondissement et de transfert à effectuer.

ii) Par l'évaluation - robuste - de ce qui détermine de grandes évolutions et les obstacles (ou risques de rupture). Celle-ci résulte à la fois de la connaissance de grands phénomènes et d'une rétrospective à grande échelle (celle des temps géologiques aussi bien que des temps historiques).

Je ne crois pas nécessaire d'insister sur cet apport de la science que chacun garde présent à l'esprit : croissance démographique, évolutions climatiques, effet de serre, raréfaction du pétrole, réduction de la diversité génétique, cycle de l'eau, etc., se mettent en perspective grâce aux travaux scientifiques et techniques. Le scientifique est celui qui maitrise les ordres de grandeur, et le temps dans la longue durée. On doit en tirer ce qu'on peut appeler le traitement des signaux lointains.

La science pourra sans doute un jour aller plus loin en analysant les couplages entre régulations, mais il faut reconnaître que l'analyse des signaux isolés sature déjà tous les moyens actuels d'élucidation et de modélisation.

iii) Par le suivi des risques à court terme et la préparation des réactions. Á une tout autre échelle du temps, le suivi en temps réel de la matérialisation du danger me parait être une des attributions croissantes et fondamentales de la science. Je prendrai l'exemple des risques naturels, mais la transposition aux autres risques de la société (explosion urbaine, épidémies, tensions nationalistes... ) est facile : on peut prévoir une crue, le passage d'un cyclone, une éruption volcanique quelques heures à l'avance : cela suffit avec les

Ce texte est extrait d'une intervention lors d'un colloque international - Maîtrise du long terme et démocratie : que environnement pour le $x_{1}{ }^{\mathrm{e}}$ siècle - qui eut lieu à

Fontevraud (France) en septembre 1997.

Ce colloque fut organisé par le ministère de

I'Environnement, l'association Germes et d'autres instances dont la revue Natures Sciences Sociétés.

Pour plus d'information s'adresser à J. Theys ou

Mme Cavagnara, Centre de prospective

et de veille scientifique, tour Pascal $B$

92055 Paris La Défense cedex 04 $\overline{\text { PAUL HenRI Bourreler }}$ Président du club Environnement de lassociation Écrin. 32, bd de Vaugirard, 75015 Paris 
moyens modernes pour détourner au dernier moment le navire de l'écueil, limiter les désastres. Le traitement des signaux d'alerte est peut-être moins glorieux que le fait d'élaborer une nouvelle théorie fondamentale et de prévoir ce qui se passera dans 100 ou 10000 ans (voir par exemple à ce sujet l'exemple des précurseurs des séismes), mais c'est sans doute un apport au moins aussi vital de la science à l'affrontement du futur.

\section{L'exercice de la responsabilité des scientifiques}

Des trois énoncés précédents sur les fondements de la responsabilité des scientifiques, je tirerai enfin, de façon non limitative, quelques considérations sur l'exercice de leurs responsabilités :

- Les quelques règles d'éthique que I'on peut appliquer ne sont pas spécifiques des scientifiques. La société a besoin d'une éthique, notamment d'une éthique de la lucidité ; celle-ci s'applique dans le cas particulier des scientifiques, comme aux politiques, aux chefs d'entreprises ou à l'homme de la rue.

- Dans une société inquiète, l'évaluation des risques en situation d'incertitude peut déboucher sur la déraison ; les scientifiques doivent élaborer une éthique de la communication appropriée (cf. vache folle).

- Je n'insiste pas sur la distinction entre science et expertise déjà longuement traitée par d'autres (par exemple Philippe Roqueplo).
- Les scientifiques ont une pratique de la controverse qui présente un intérêt par son instrumentation (forums tenus par la communauté mondiale, etc.) mais il ne faut pas se tromper : la sociologie de la science montre qu'il n'y a rien d'absolument spécifique dans les jeux des intérêts et des pouvoirs. La science apporte peut-être une démonstration particulièrement frappante de la fertilité de la dialectique, de l'élaboration et des remises en question de dogmes et théories qui paraissent bien établis ; elle doit montrer l'exemple dans l'acceptation permanente d'un pluralisme raisonnable.

- Dans ce cadre on devrait s'intéresser à l'utopie scientifique qui a trouvé une expression dans la science fiction, mais qui, dans une navigation au juger (détection des obstacles à courte distance), peut aussi aider dans l'urgence à trouver les solutions de fortune. - II y aurait aussi à réfléchir sur le mauvais couplage des sciences de l'homme et de la société, le droit de la responsabilité constitue, par exemple, une discipline qui mérite une priorité.

En conclusion, sans faire de paradoxe, je crois que si la science est apte à fournir quelques précieux instruments de navigation, le problème est que ces instruments ne sont peut-être pas exactement ceux qu'on lui demande ni ceux que certains scientifiques aimeraient fournir ; n'y a-t-il pas un malentendu ? Je le crains, comme je me méfie d'opérations telles que celle qui consiste à interroger les scientifiques sur la priorité des recherches à faire. II faudrait peut-être commencer par une révision plus fondamentale du - cahier des charges - de la science, dans sa convention implicite avec la société. 